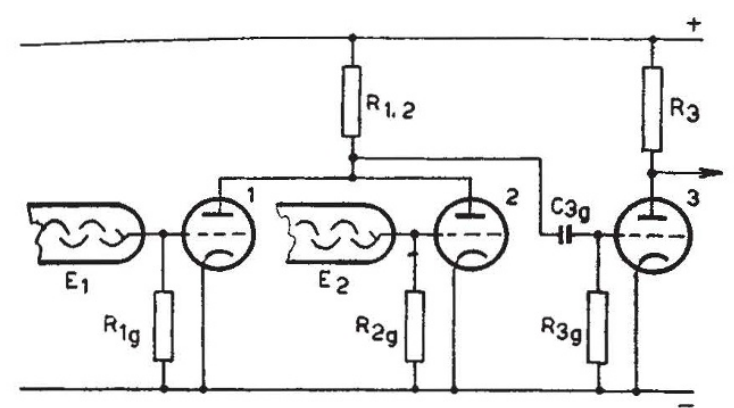

input pulse, but so as to respond to pulses of at least $2 \mathrm{~V}$., corresponding to coincidences.

The resolving power so far achieved is about $5 \times 10^{-9}$ sec. The determination of this quantity was carried out-besides the usual method of counting random coincidences-by a direct method. The impulses of the same multiplier tube are fed into both inputs of the device, so that each impulse is recorded as an 'artificial coincidence'. If one of the leads connecting the multiplier to one of the inputs is increased by a length $\Delta l$, this side of the device will be reached by the impulse $\Delta t=\Delta l / c$ sec. later; for example, $1.5 \mathrm{~m}$. corresponding to the resolving power given above. Because of this time-lag the amplitudes of the artificial coincidences decrease, and at a critical length they fade out entirely. By increasing the length of the other lead, too, the coincidences are made to reappear, demonstrating that fading-out of the coincidences was a consequence of the time-lag caused by the difference in length of the leads.

The device was designed chiefly for investigations in nuclear physics. At present, experiments similar to those of Bothe ${ }^{2}$ are going on in the ultra-violet and visible region.

Further details will be published elsewhere.

$$
\text { Z. BAY }
$$

G. PAPP

Institute of Atomic Physics,

University of Technical and

Economic Sciences,

Budapest.

July 25.

'Papp, G. (unpublished).

Bothe, Z. Phys., 37, 547 (1926).

\section{Electron Multiplier Tube of Large Effective Cathode Surface Area}

Electron multiplier tubes have been used in this Laboratory for particle counting ${ }^{1}$, and their extremely short impulse period ${ }^{2}$ makes them very useful research tools'. One disadvantage of these tubes is their small effective surface.

One method for increasing the effective surface is the following : the electron-optical image of a cathode of large surface area is projected on the first multiplying electrode of a multiplier tube of the usual dimensions. In this way a tube similar to the partition. type multiplier of Zworykin and Rajchman ${ }^{4}$ was designed. The electrons are emitted by a cathode of spherical shell shape, and the image of the surface of the cathode is projected on the first multiplying electrode by an electrostatic lens. The cathode subtends an angle of $90^{\circ}$ viewed from the focus of the lens. In this manner an effective surface of $100 \mathrm{~cm} .^{2}$ can easily be obtained.
The centre of curvature of the cathode is at the focus of the lens; the transit time of the electrons between cathode and first multiplying surface is very nearly the same for all electrons, regardless of their point of origin. This is most important in coincidence devices of very high resolving power.

The dimensions of the lens electrodes and the potentials thereon can be adjusted so that practically every electron emitted by any point of the cathode can reach the first multiplying stage without being captured by intermediate electrodes or repelled to the cathode.

In the experimental tubes, electrons could be released from well-defined points of the cathode. Some of the tubes were enlarged models, having electrodes covered by a thin layer of fluorescent material. In this way the incidence of the electrons was made visible. This method was very useful, especially in the first qualitative experiments.

Another possible way of increasing the effective surface of the electron multiplier tubes is to increase the length of all electrodes of a circular-type multiplier ${ }^{4}$. This method, however, would increase the eapacity of the collector plate, which is a disadvantage from the point of view of the impulse period of the tube and the amplitude of the voltage pulse.

The development of the tube for the purpose of researches on cosmic radiation is going on.

Tungsram Research Laboratory,

P. S. Faragó

Ujpest 4, near Budapest. July 25.

${ }^{1}$ Bay, Z., Z. Phys., 117, 227 (1941)

Papp, G. (unpublished).

B Bay, Z., and Papp, G. (see preceding letter).

- Zworykin, V. K., and Rajchman, J. A., Proc. Inst. Rad. Eng., 27, $558(1939)$.

\section{Variation of Thermo-electric Power of Electronic Semi-Conductors with Vapour Pressure}

When the principle of mass action is applied to explain the effect on the electrical properties of certain electronic semi-conductors having an ionic lattice structure, produced by varying the vapour pressure of one of the constituents of the substance, it is found that

$$
n_{f}=K P_{v^{1 / n}},
$$

where $n_{f}$ is the concentration of charge carriers, which may be free electrons or holes, $K$ is an equilibrium constant, $P_{v}$ is the vapour pressure, and $n$ is a constant, negative for excess and positive for defect semi-conductors, which can be deduced from the chemical structure of the substance.

If this equation is introduced into the usual freeelectron theory for the thermo-electric power of semiconductors $^{1}$, equations may be obtained corresponding to the empirical equation

$$
\frac{d E}{d T} \pm \text { const. }=-C_{T} \log P_{v},
$$

where $d E / d T$ is the thermo-electric power, taken as negative for excess and positive for defect semiconductors, and $C_{T}$ is a constant for a particular substance. From the theory $C_{T}$ may be identified with $k /|e| n$, where $k$ is Boltzmann's constant and $e$ is the electronic charge, and a value for $n$ thereby obtained.

Little experimental work has been performed on this subject except for a few spot readings by Wagner and his co-workers ${ }^{2,3,4}$, made to test Wagner's 